\title{
272 A NOVEL BISPECIFIC MACROPHAGE ENGAGER ANTIBODY (BIME) DESIGNED FOR CANCER IMMUNOTHERAPY
}

Hongtao Lu*, Dawei Sun, Xiaofeng Niu, Yanan Geng, Jing Wang, Haixia Jiang, Rui Gao, Zhihao Wu, Yangsheng Qiu. Elpiscience Biopharmaceuticals, Shanghai, China

Background Anti-CD3-based bispecific T cell engagers (BiTE) showed limited clinical efficacy in solid tumors. This is partly due to the difficulty and scarcity of $\mathrm{T}$ cells infiltrating into tumor microenvironment (TME). Macrophages are major component of immune cell infiltrate in the TME and can constitute up to $50 \%$ of a solid tumor mass. Signal regulatory protein- $\alpha(\operatorname{SIRP} \alpha)$ is a major myeloid cell inhibitory receptor that engages the "don't eat me" signal from CD47 expressed on tumors. Similar to BITE where T cells are activated by the CD3 antibody, we constructed a novel bispecific macrophage engager (BiME) where macrophage is activated by a SIRP $\alpha$ inhibitory antibody that is directed to a particular tumor via the tumor associated antigen (TAA) antibody, resulting in phagocytosis of the tumor. Previously, we have developed a human SIRP $\alpha$ monoclonal antibody called ES004-B4, that blocks CD47 binding. ES004 greatly augments antibody dependent cellular phagocytosis killing of cancer cell lines. We utilize ES004 to make a BiME called ES028 where the SIRP $\alpha$ antibody is linked to a Claudin18.2 antibody, targeting claudin18.2-expressing cancers like gastric cancer.

Methods Through Elpiscience BiME platform, we have generated a panel of anti-Claudin18.2/SIRP $\alpha$ bispecific antibodies, including different anti-Claudin18.2 arm and anti-SIRP $\alpha$ arm positions, ratios and $\operatorname{IgG}$ isotypes. The binding and blocking ability of these bispecific antibodies were evaluated by ELISA and FACS. In vitro function activity was determined by phagocytosis assay using human monocyte derived macrophage and mouse bone marrow derived macrophage. In vivo anti-tumor efficacy was investigated in a syngeneic tumor model with hSIRP $\alpha$ knock-in mice.

Results We demonstrate that an anti-Claudin18.2/SIRP $\alpha$ bispecific antibody ES028 exhibited super anti-cancer effects, with improved phagocytosis of cancer in vitro and extended survival of claudin18.2-expressing tumor burden mice. In the syngeneic model, ES028 showed almost 100\% tumor growth inhibition in the SIRP $\alpha$ knock-in models without causing cytokine storm. ES028 could not induce phagocytosis of Claudin18.2 negative cells, proving its specificity and selectivity.

Conclusions We have developed a bispecific macrophage engager (BiME) platform that is capable of activating phagocytosis to kill cancer cells. A number of preclinical programs are ongoing to design specific BiME for particular tumor indication. Our SIRP $\alpha$-based macrophage engager ES028 is the first to have reached the pre-clinical stage with a demonstrated favorable safety profile and promising therapeutic efficacy. Taken together, these results indicate that the bi-specific macrophage engager platform is feasible and could be a powerful weapon in the battle towards the elimination of cancers.

http://dx.doi.org/10.1136/jitc-2021-SITC2021.272 